\title{
Correction to: An analysis of 11.3 million screening tests examining the association between recall and cancer detection rates in the English NHS breast cancer screening programme
}

\author{
R. G. Blanks ${ }^{1} \cdot$ R. M. Given-Wilson ${ }^{2}$ - S. L. Cohen ${ }^{3} \cdot$ J. Patnick $^{1}$ • R. J. Alison ${ }^{1} \cdot$ M. G. Wallis ${ }^{4}$
}

(C) European Society of Radiology 2019/Published online: 5 July 2019

\section{Correction to: European Radiology}

https://doi.org/10.1007/s00330-018-5957-2

The original version of this article, published on 04 February 2019, unfortunately contained a mistake. The following correction has therefore been made in the original: The presentation of Fig. 2 was incorrect. The corrected figure is given below.

The online version of the original article can be found at https://doi.org/ $10.1007 / \mathrm{s} 00330-018-5957-2$

\section{R. G. Blanks}

roger.blanks@ndph.ox.ac.uk

1 Cancer Epidemiology Unit, Nuffield Department of Population Health, Oxford University, Oxford, UK

2 Department of Radiology, St Georges University Hospital Foundation Trust, London, UK

3 Public Health England, London, UK

4 Cambridge Breast Unit and NIHR Cambridge Biomedical Research Centre, Cambridge University Hospitals NHS Trust, Cambridge, UK 
a

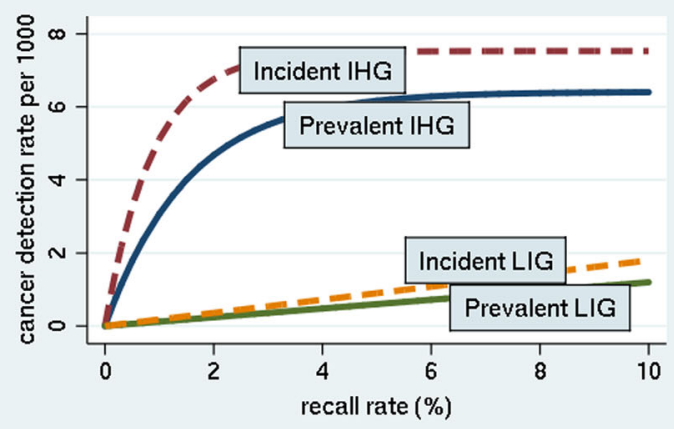

C

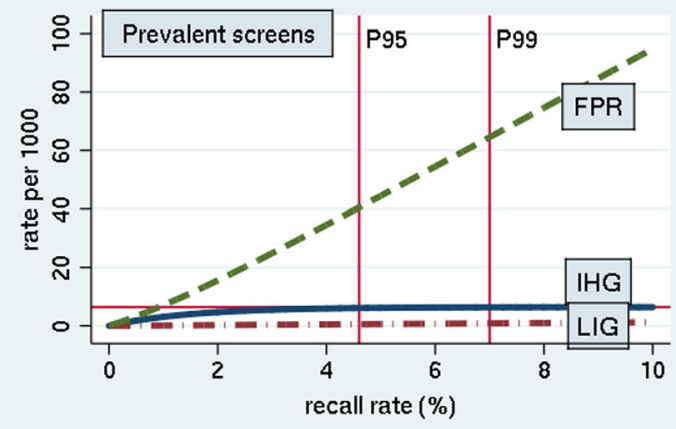

Fig. 2 Modelled cancer detection rate per 1000 against recall rate (a) and false positive recall rate (b) for IHG and LIG at incident and prevalent screens. Modelled false positive recall (FPR), IHG and LIG per 1000 for b

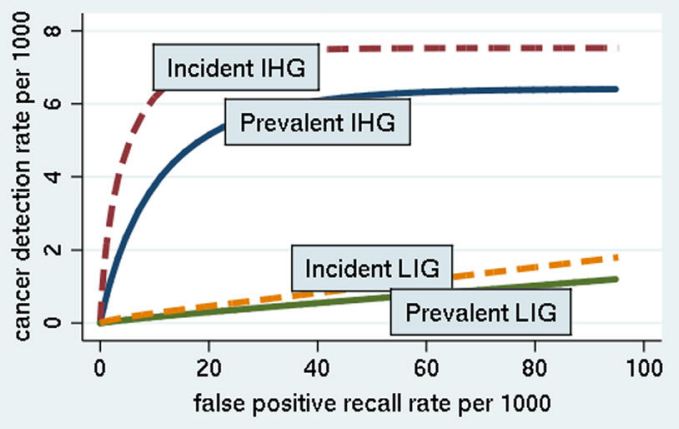

d

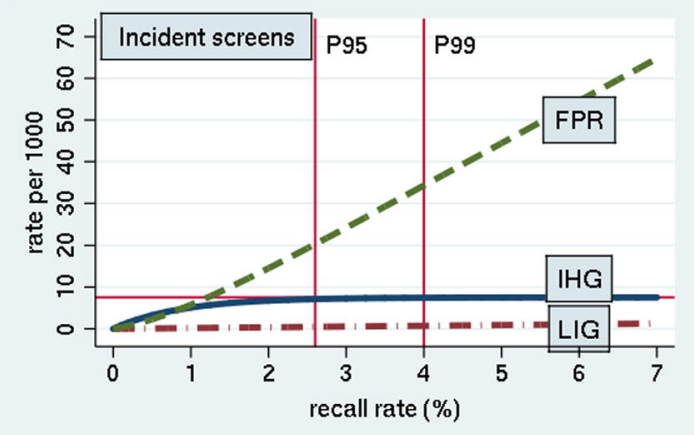

prevalent screens (c) with P95 and P99 recall rate values and same for incident screens $(\mathbf{d})$

Publisher's note Springer Nature remains neutral with regard to jurisdictional claims in published maps and institutional affiliations. 